\title{
Performance Improvement of Conventional Type Electrostatic Oil Filter with Reusable Filter Element*
}

\author{
Hideki YANADA**, Yamato KATO***
}

This paper deals with an improvement of filtration speed of an electrostatic oil filter. Commercially available electrostatic oil filters use a dielectric material such as paper as filter element. The filter element needs to be regularly exchanged to keep the filter performance and becomes waste after use. In order to minimize waste, reusable filter elements made of stainless steel are used in an electrostatic oil filter proposed. The effects of the configurations of the electrodes and filter elements on the electric and flow fields in the filter are numerically examined and three types of filter configuration are made based on the numerical simulation results. The effect of the filter configuration on the filtration speed is examined experimentally. The effect of the shape of the filter element on the filtration speed is also examined using one type of filter. It is shown that the filtration speed can be increased by making the flow velocity distribution in the filter uniform and an optimal filter configuration is proposed.

Key words : Filter, Contamination, Electrostatic filter, Lubricating oil, Reusable filter element, Flow field, Electric field, Oil saving

\section{Introduction}

An electrostatic oil filter can remove submicrometer-sized contaminants such as the oxidation products of additives from oils. By virtue of this characteristic, it has contributed to lengthening the lives of lubricating oils and to decreasing waste oil as well as failures of machines including hydraulic systems ${ }^{1)}$. The filtration principle of the electrostatic oil filter is based on the fact that solid particles in liquid are usually charged positive or negative due to the preferential adsorption of ions in liquid and the dissociation of dissociative groups on the surfaces of the particles ${ }^{2}$. By applying a high DC voltage between the electrodes through which oil flows, charged contaminants in the oil are attracted towards and captured on the surfaces of the electrodes and a filter element that is inserted between the electrodes and is made of dielectric material like paper. The role of the filter element is to increase capturing area and to capture conductive contaminants like metallic wear debris, which cannot be captured on the electrode surfaces.

Commercially available electrostatic oil filters utilize a dielectric material such as paper as the material of the filter

\footnotetext{
*Manuscript received June 13, 2009

** Toyohashi University of Technology

(1-1, Hibarigaoka, Tempaku-cho, Toyohashi, Japan)

E-mail : yanada@mech.tutac.jp

****Panasonic Ecology Systems Co., Ltd.

(4017, Shimonakata, Takaki-cho, Kasugai, Japan)
}

element ${ }^{1)}$. The filter element has to be regularly exchanged to a new one to keep the filter performance and a used filter element becomes waste. Nowadays, reduction of waste is an important issue in the world. If filter elements made of metal such as stainless steel are used, they can be semipermanently used by washing them periodically. For this case, the washing liquid may become waste after the use during some period of time. It is not easy to significantly decrease waste but the semipermanent use of the filter elements can decrease not only the quantity of waste but also the cost.

Yanada and his colleagues made a cooperative research project to develop a high-performance electrostatic oil filter using reusable filter elements in cooperation with two enterprises under the financial support by the Ministry of Economy, Trade and Industry, Japan from 2004 until 2005². Developed electrostatic oil filters have a faster filtration speed than the ones available on the market and are now commercially available in Japan. During the project period, the effects of mechanical factors such as the configuration of the filter element were partly examined but were not fully done. There is still room for improving the filtration speed of the electrostatic oil filter. The increase in the filtration speed contributes to the decrease in the filtration time or to the downsizing of the filter.

In the electrostatic oil filter developed in 3), oil crosses the filter elements and electrodes but does not flow in parallel with them. Such a configuration was designed based on the result obtained in a fundamental investigation using a single fiber model ${ }^{4)}$. However, experimental verification has not 
been done using a prototype of the electrostatic oil filter. In this investigation, the effect of the configuration of the filter element on the filtration speed is examined experimentally and numerical simulation of electrostatic field in the filter is conducted to discuss the results. In addition, in order to find out an optimal configuration of the electrostatic oil filter, three types of electrostatic filter having different configurations are made and filtration speed is compared among the three.

\section{Experimental Apparatus and Method}

\subsection{Configuration of Electrostatic Oil Filter}

Figure 1 shows a schematic of the electrostatic oil filter developed in the abovementioned research project $t^{3)}$. The filter of this configuration is called Type 1 in this paper. The oil flows into the upper space of the filter from the inlet port, is divided into two in the upper space and then flows into the main part (filtration chamber) through the slots machined at the side edges of the base plate of the earth electrodes. The main parts of the filter consist of the high voltage electrodes, earth electrodes, filter elements, insulating plates made of polycarbonate. The filter casing is made of stainless steel square pipe and its inner walls take roles as earth electrodes.

The electrodes and the backplates of the filter elements are made of perforated stainless steel plates, of which open area ratio is $40.2 \%$, in order for the oil to cross the electrodes and filter elements as shown in Fig.4(a). A stainless mesh bent in wavy form is attached to one side of each backplate of the filter element to increase the capturing area. Six filter elements can be inserted at the maximum but, in this investigation, four filter elements were inserted as shown in Fig. 1. Grooves are regularly machined on the surfaces of the insulating side plates (10mm thick, not shown) to fix the positions of the electrodes and filter elements. Oil flows out of the outlet port at the middle of the bottom of the filtration chamber. The volume occupied by the oil in the filter is $2.0 \times$ $10^{-3} \mathrm{~m}^{3}$.

The direction of each filter element can be changed according to the electrification polarity of contaminants and are alternately changed as shown in Fig. 1. For example, when the polarity of the high voltage electrodes is negative and the majority of the contaminants are charged positive, the desirable directions of the filter elements are those shown in Fig. 1. In this case, negative charges are induced on the outer surfaces of the mesh of the filter elements. Therefore, positively charged contaminants can be captured on the outer surfaces of the meshes as well as on the negative electrodes.

Numerical simulation of flow field, part of which results are shown in Section 3, showed that for the configuration shown in Fig. 1, there are regions where oil stagnates and the stagnant regions occupy a relatively large space. This means that all the space in the filtration chamber cannot be efficiently utilized. In order to decrease the stagnant regions and to make the flow velocity distribution uniform, numerical

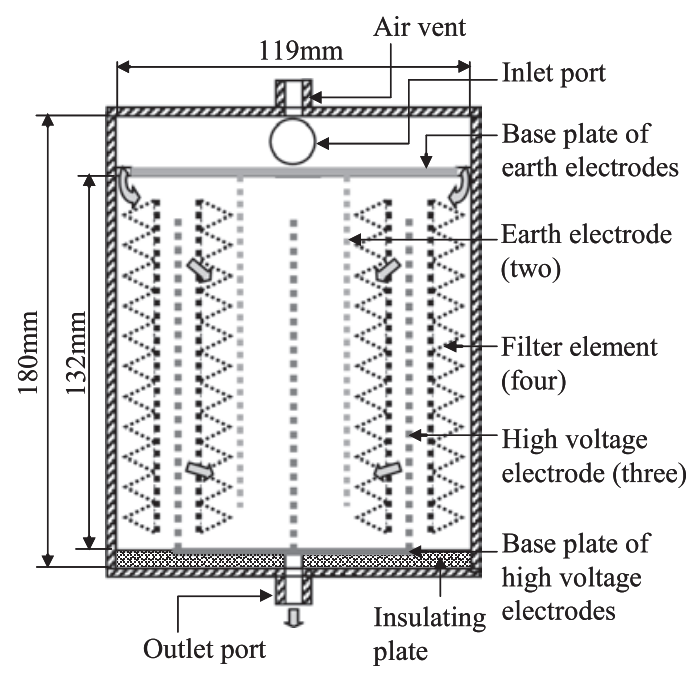

Fig. 1 Schematic cross-sectional view of Type 1 filter

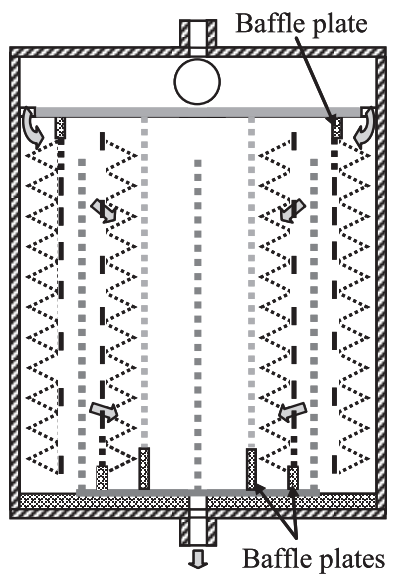

Fig. 2 Schematic cross-sectional view of Type 2 filter

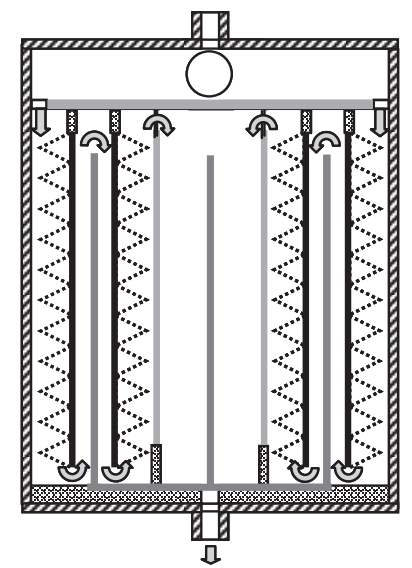

Fig. 3 Schematic cross-sectional view of Type 3 filter 


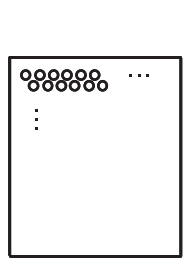

(a) Type 1

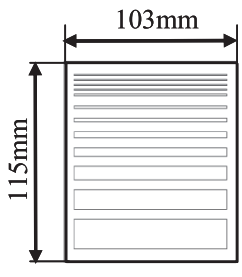

(b) Type 2

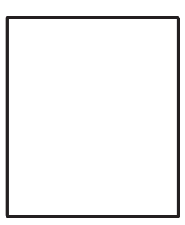

(c) Type 3
Fig. 4 Backplates of filter elements

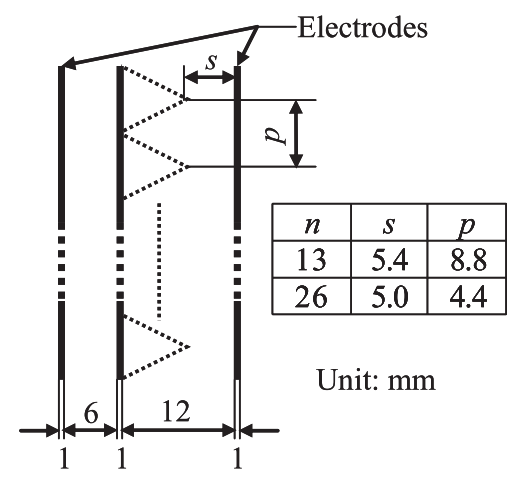

Fig. 5 Arrangement of electrodes and filter element

simulations of the flow field were conducted for various shapes of the openings of the backplates of the filter elements. An alternative type of the filter is shown in Fig. 2 and is called Type 2. The shape of the openings of the backplates of the filter elements is rectangle in Type 2 and the area of each opening is varied exponentially in one direction as shown in Fig. 4(b). The open area ratio of the backplates is the same as that of Type 1. The outermost filter elements are inserted such that the area of the opening is increased from the top to the bottom and the top and bottom of the next filter elements are reversed. In addition, baffle plates made of polycarbonate are attached. By doing so, the flow velocity distribution becomes relatively uniform in Type 2 filter as can be seen in Section 3.

Another type of electrostatic oil filter, Type 3 , is shown in Fig. 3. The electrodes and the backplates of the filter elements are made of solid stainless plates (Fig. 4(c)) and oil flows in parallel with those. However, also for this case, oil crosses the stainless meshes because they are bent in wavy form. For Type 3, there is no stagnant region and local flow velocity is increased due to decreased flow cross-sectional area compared with Type 2 and Type 3 .

Figure 5 shows the arrangement of the electrodes and filter elements. The distance between the crests of the wavy mesh and their neighboring electrode is slightly decreased with increasing number, $n$, of the folds (or crests) of the mesh. Three types of filter mesh of $n=0,13,26$, of which size is 100 , were used. In addition, the filter elements, of which number of the folds is 26 , with different mesh sizes $(100,200$, 400 mesh) were made. The wire diameter is 0.080, 0.040 and $0.020 \mathrm{~mm}$ for 100, 200, and $400 \mathrm{mesh}$, respectively. Those effects on the filtration speed were examined using Type 1 filter. The open area ratio of the perforated plates used for the electrodes and the backplates of the filter elements was fixed to $40.2 \%$.

\subsection{Filtration Experiment}

Six types of artificially contaminated lubricating oils were used for filtration experiments. A test particle (JIS 1st kind, class 11, Kanto loam, median diameter $=1.6-2.3 \mu \mathrm{m})^{5)}$ was mixed at a concentration of $0.5 \mathrm{~g} / 1$ into the oils. In general, conductive contaminants such as metallic wear debris and non-conductive ones such as oxidation products of additives are both included in lubricating oils. The former can be captured only on the filter elements and the latter on both the filter elements and electrodes. In this investigation, only the non-conductive particles were used as contaminants.

In Table 1 , the values of the conductivity and viscosity of the test oils at a temperature of $313 \mathrm{~K}$ are shown. Oils 1 to 4 are hydraulic fluids and Oils 5 and 6 are metal working fluids. The "polarity" written in the last column of Table 1 indicates the electrification polarity of the test particles mixed in the oils. The particles were charged positive in Oils 1 to 3 and charged negative in Oils 5 and 6 . The majority of the particles were charged positive and partly negative in Oil 4. When making filtration experiments using Oils 5 and 6, the directions of the filter elements were changed from those shown in Figs. 1 to 3.

A schematic of the experimental apparatus used is shown in Fig. 6. Artificially contaminated oil in reservoir (1) is fed into the filter (4) by a gear pump (3) and then returns to the reservoir. The oil in the reservoir is stirred by a magnetic stirrer (2) to avoid the gravitational sedimentation of the particles. The temperature of the oil was fixed at 313K during the experiment using a thermometer (6), a heater (7)

Table 1 Physical properties of test oils at 313K ( $\sigma$ : conductivity, $\mu$ : viscosity)

\begin{tabular}{|c|c|c|c|}
\hline Oil No. & $\sigma[\mathrm{S} / \mathrm{m}]$ & $\mu[\mathrm{Pa} \cdot \mathrm{s}]$ & Polarity \\
\hline 1 & $5.31 \times 10^{-12}$ & $9.97 \times 10^{-3}$ & + \\
\hline 2 & $1.35 \times 10^{-10}$ & $19.4 \times 10^{-3}$ & + \\
\hline 3 & $1.24 \times 10^{-10}$ & $39.4 \times 10^{-3}$ & + \\
\hline 4 & $4.71 \times 10^{-13}$ & $37.9 \times 10^{-3}$ & $+/-$ \\
\hline 5 & $3.48 \times 10^{-10}$ & $61.2 \times 10^{-3}$ & - \\
\hline 6 & $4.68 \times 10^{-8}$ & $50.9 \times 10^{-3}$ & - \\
\hline
\end{tabular}




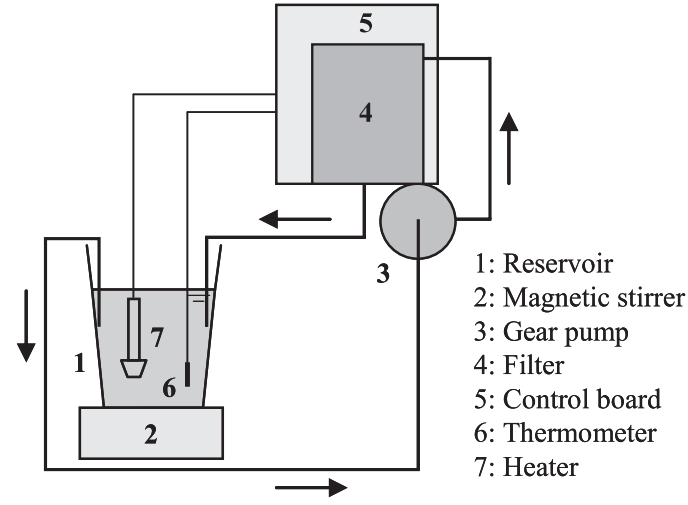

Fig. 6 Schematic of experimental apparatus

and a thermoregulator. A high voltage DC power supply and a thermoregulator are incorporated in the control board (5). In the filtration experiments, the applied voltage was fixed at $-10 \mathrm{kV}$. The flow rate was $15 \times 10^{-6} \mathrm{~m}^{3} / \mathrm{s}$. The volume of test oil was $5 \times 10^{-3} \mathrm{~m}^{3}$. About $20 \times 10^{-6} \mathrm{~m}^{3}$ of oil was sampled from the reservoir at specified times during the filtration experiment to evaluate the variation of the concentration of the particles in the oil with time. The mass concentration of the particles included in the sampled oil was measured by filtering it together with enough volume of hexan, a solvent, using membrane filters having a pore size of $0.8 \mu \mathrm{m}^{6}$.

\section{Results and Discussion}

\subsection{Effect of Shape of Filter Element}

Filtration experiments that examine the effect of the number of the folds of the stainless mesh were carried out using Oils 2 and 3. Experimental results of Oil 3 are shown in Fig. 7, of which ordinate and abscissa indicate the particle concentration and the filtration time, respectively. 100\% indicates the initial concentration, $0.5 \mathrm{~g} / 1$. As can be seen from Fig. 7, the speed of the decrease in the particle concentration, i.e., the filtration speed was increased with increasing number of the folds of the mesh. Similar results were obtained for Oil 2. The effect of the mesh size on the filtration speed was examined using three sizes of mesh (100, 200 and 400 mesh) but the filtration speed was hardly affected by the mesh size. This means that 100 mesh is the best as filter elements from the standpoints of strength and cost of the filter elements.

Numerical simulations of the electrostatic field in the filter were made using ANSYS to discuss those results. In the simulations, the mesh and its backplate were approximated as two-dimensional because an exact geometric modeling of the mesh is difficult. Figure 8 shows the approximated geometric models of the filter elements with different

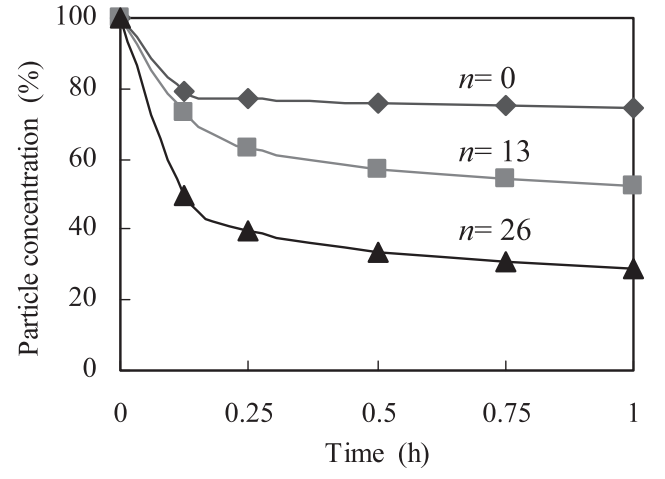

Fig. 7 Effect of number of folds of mesh on filtration speed (100mesh, Oil 3)

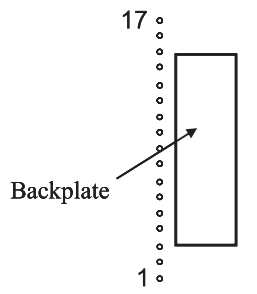

(a) $n=0$

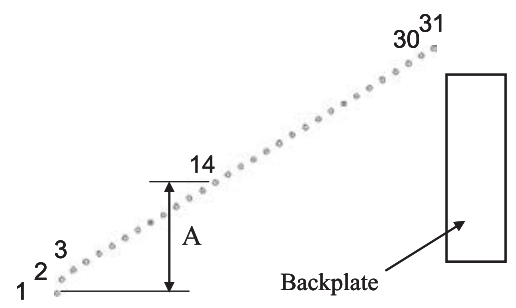

(b) $n=13$

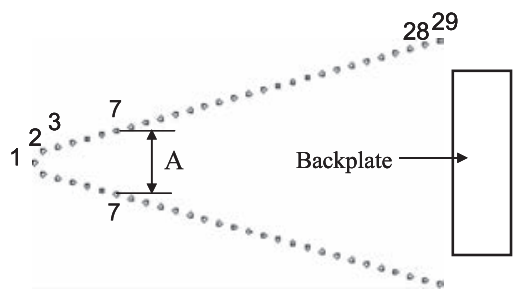

(c) $n=26$

Fig. 8 Two-dimensional model of filter element (100 mesh)

numbers of the fold of mesh. For $n=13$ and 26, the curvature at the bottom of the mesh was neglected for simplicity and, therefore, the number of the wires is different between the two cases. The electric field strength near the backplate becomes very low and such an approximation has no significant influence on the electric field strength distribution. In the models shown in Fig. 8, the element wires of the mesh and the backplate look separated each other but, in practice, they are electrically connected. Therefore, a boundary condition that they have a same electric potential was given in the simulations. The meaning of the region $\mathrm{A}$ is explained 

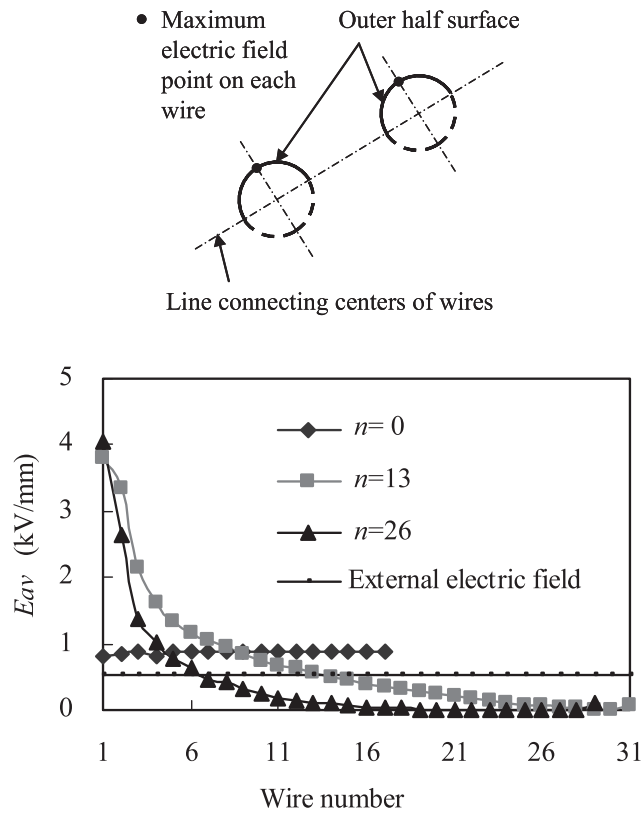

Fig. 9 Electric field strength distribution on mesh surface (100 mesh)

later.

Figure 9 shows electric field strength distributions on the mesh surface. The ordinate stands for the electric field strength averaged over the outer half surface, $E_{a v}$, of each wire, which is shown by solid semicircles in the upper part of Fig. 9, and the abscissa stands for the wire number shown in Fig. 8. The maximum electric field strength on each wire appears at the position where a radius line intersects at right angles to the line connecting the center of each wire. The electric field strength on the inner half surface is low and is not very effective to capture contaminants. Therefore, the inward half of the wire was omitted from the calculation of the average value of the electric field strength.

For the wavy mesh ( $n=13$ and 26), the spacing between the crests of the mesh and their neighboring electrode is short and the electric field strengths at and near the crests become much higher than the electric field strength for $n=0$. On the other hand, the electric field strengths on the wires near the backplate become lower than the applied external electric field strength $\left(E_{e x}=0.526 \mathrm{kV} / \mathrm{mm}\right)$, which is defined as the applied voltage $(10 \mathrm{kV})$ divided by the distance between the electrodes $(19 \mathrm{~mm})$. It is considered that the high electric field formed at and near the crests contributes to the increase in the filtration speed.

The electric field strength on each wire is higher for $n=13$ than for $n=26$ except for no. 1 wire. The total number of the wires on which the electric field strength is nearly equal to or higher than $E_{\text {ex }}$ is almost the same for $n=13$ and $n=26$ (14 wires for $n=13$ and 13 wires for $n=26$, which are the wires in

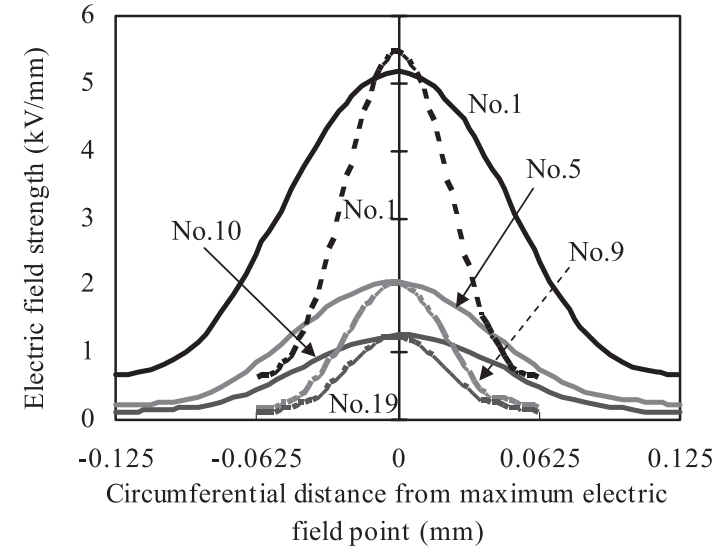

Fig. 10 Electric field strength distribution around wire ( $n=13$, solid: 100 mesh, dotted: 200 mesh)

the region 'A' shown in Fig. 8). It seems that the filter element of $n=26$ has no advantage over that of $n=13$ from the standpoint of the electric field strength on the element surface. Taking the results of the filtration experiments into account, it is considered that a narrower width of the high electric field area 'A' may contribute to an efficient capture of contaminants because of the decreased distance between adjacent wires in the direction perpendicular to the flow.

Figure 9 also shows that the electric field strength is lower than the applied external electric filed strength over the majority of the mesh surface, and that the low electric field area is increased with increasing number of the folds. These suggest that the filtration speed may be increased more by devising the configuration of the filter element.

Electric field strength distributions on the wire surfaces are shown in Fig. 10, in which simulation results for 100 mesh and 200 mesh are compared. Three wires that are situated at corresponding positions were selected from each mesh size (no. 1, 5, 9 for 100 mesh and no. 1, 9, 19 for 200 mesh). The abscissa indicates the circumferential distance measured from the point where the maximum electric field strength appears on each wire. Except for no.1 wire, both the maximum and average electric field strengths on each corresponding wire are almost the same. In addition, the total extent of high electric field areas on wire surfaces is almost the same between 100 and 200 mesh. The same can be said to 400-mesh filter element. Therefore, it is considered that the filtration speed is not affected by the mesh size.

\subsection{Effect of Filter Configuration}

Figure 11 shows the profiles of the lateral component, $u$, of the flow velocity on the four planes A, B, C, D shown in Fig. 11(a). The planes $\mathrm{A}$ and D are situated at the crests of the wavy mesh and planes $\mathrm{B}$ and $\mathrm{C}$ at the middle of the outermost high voltage electrode and the backplates of its 


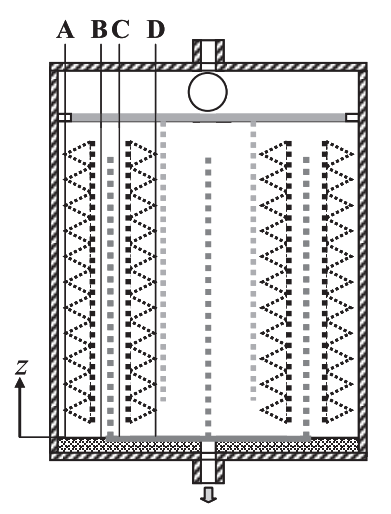

(a) Planes for comparison of velocity profile

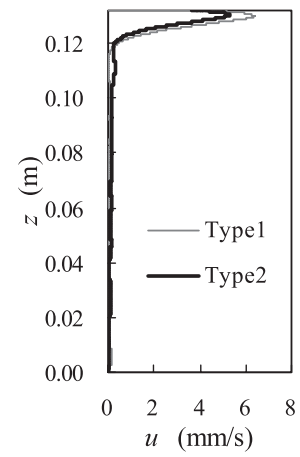

(b) On plane A

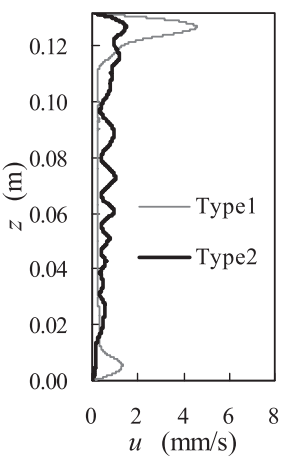

(d) On plane C
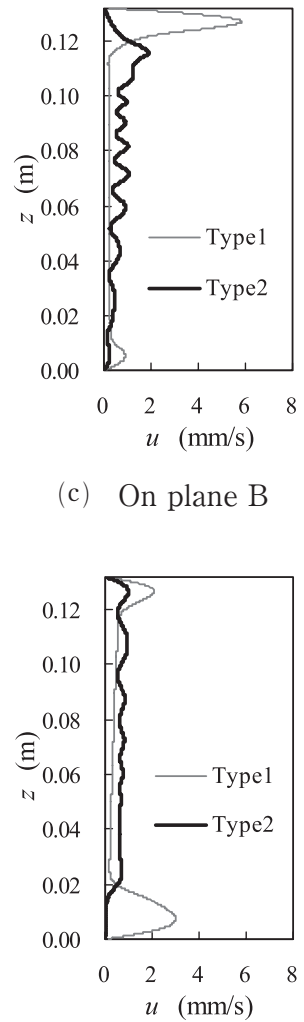

(e) On plane D (c) On plane B

Fig. 11 Profile of lateral velocity component in filtration chamber

neighboring two filter elements. The velocity profiles were obtained by numerical simulation using the software CFX5.0. In the simulation, the wavy mesh was neglected because of the difficulty of its geometric modeling. The velocity profiles are not varied largely in the direction perpendicular to the sheet.

For Type 1 filter, the lateral velocity component on plane $\mathrm{A}$ is extremely large immediately below the base plate of the earth electrodes and the same can be said also on plane B, while, for Type 2 filter, the velocity profile becomes relatively uniform on plane $\mathrm{B}$. The numerical simulations showed that the velocity distribution cannot become uniform when

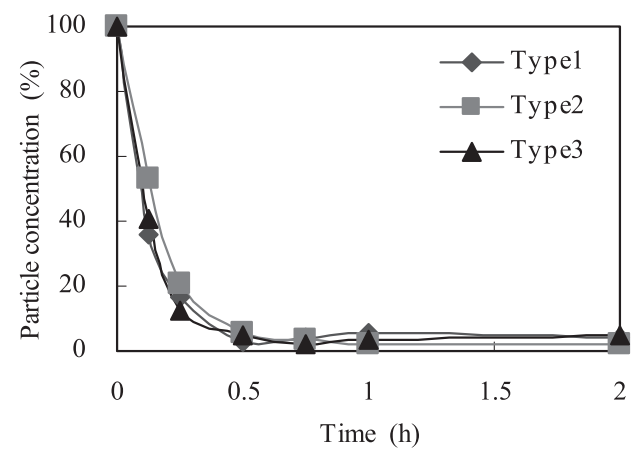

(a) Oil 1

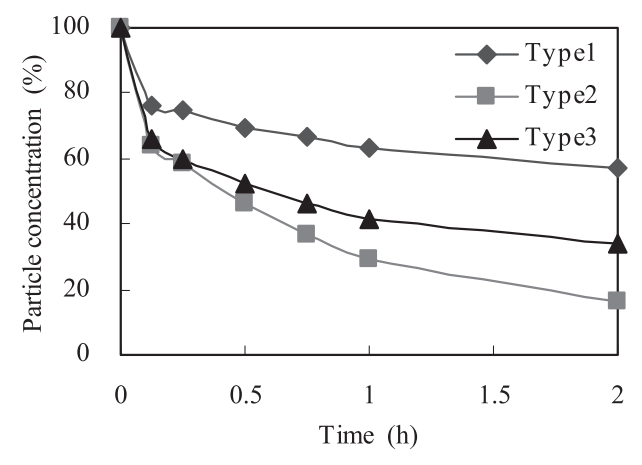

(b) Oil 2

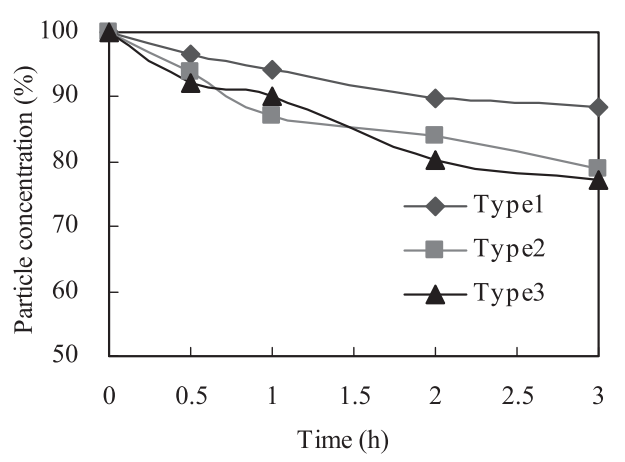

(c) Oil 6

Fig. 12 Effect of filter configuration on filtration speed $(n=26)$

perforated plates are used as the backplates of the filter elements but can be uniform by changing exponentially the opening area of the backplates and by inserting the baffle plates. Though not shown in the figure, the velocity profile is not made uniform only by inserting the baffle plates into Type 1 filter.

Comparison of the filtration speed among three types of filter was made and experimental results are shown in Fig. 12. For relatively easily purified oils such as Oil 1 , the filtration speed was not affected by the filter configuration as shown in Fig. 12(a). Similar results were obtained from Oils 4 and 5. However, for Oils 2 and 6, the filtration speed was significantly increased by changing the filter configuration 
from Type 1 to Type 2 as shown in Figs. 12(b) and (c). Because Oil 6 was not easily purified compared to the other test oils, the filtration experiment was conducted for a longer time, 3 hours. As shown in Fig. 12(c), when Type 2 is used, the time to decrease the particle concentration to a certain level, e.g. $90 \%$, can be reduced to shorter than the half the time needed when Type 1 is used. There is no difference in the basic configuration between Type 1 and Type 2 and only the uniformity of the flow velocity is different.

The filtration speed can be increased by changing the filter configuration from Type 1 to Type 3 as well. The difference in the filtration speed between Type 1 and Type 3 is caused not only by the difference in the uniformity of the flow velocity but also by the difference in filter configuration. It is not easy to separate the effects of the two factors. However, it can be concluded from the results shown in Fig. 12 that the filtration speed can be increased by making the flow velocity distribution uniform and that Type 2 is better than Type 3 .

\section{Conclusion}

The effects of the configuration of filter element on the filtration speed of electrostatic oil filter were examined experimentally and the experimental results were discussed based on the numerical simulation results of the electrostatic field in the filter chamber. In addition, filtration experiments were conducted using three types of electrostatic oil filter. The following conclusions can be drawn from the present investigation:

1. The filtration speed is increased with increasing number of the folds of the wavy mesh of filter element.

2. The mesh size hardly affects the filtration speed.

3. Uniformity of the flow velocity distribution in filtration chamber can increase the filtration speed.

4. Type 2 filter is the best among the three filters used.

The present investigation shows that a simple modification of the filter configuration can increase the filtration speed. It may be increased more by devising the configuration of the filter element. Further investigation is necessary.

\section{Acknowledgements}

The authors would like to express their gratitude to T. Okamoto for his help in part of the numerical simulation.

\section{References}

1 ) Sasaki, A., Sasaoka, M., Tobisu, T., Uchiyama, S., Sasaki, T.: The Use of Electrostatic Liquid Cleaning for Contamination Control of Hydraulic Oil, Lubrication Engineering, 44-3, 251/256 (1988)

2) Kitahara, A., Watanabe, A.: Electrical Phenomena at Interfaces. Fundamentals, Measurements, and Applications, Marcel Dekker, Inc. (1984)

3 ) Kishi, K., Yanada, H., et al: Development of a HighPerformance Electrostatic Oil Filter, Proc. 82nd Annual Meeting of Kansai Branch, Japan Soc. Mech. Engrs., 074-1, 11 (room12), (2007) (in Japanese)

4) Yanada, H., Takikawa, Y: Experimental Approach to Electrostatic Liquid Filtration using a Single Fibre Model, Part 2 : Effects of Mechanical Factors, Proc. Instn. Mech. Engrs, Part C: J. Mech. Eng. Sci., 208-C6, 387/393 (1994)

5 ) Japanese Industrial Standard, Test Powders and Test Particles, JIS Z8901, (1995)

6 ) ASTM Standards, Standard Test Method for Insoluble Contamination of Hydraulic Fluids by Gravimetric Analysis, F313, (1983) 